\title{
Zubehör für Hörsysteme Patienteninformation
}

\section{Hörassistierende Techniken | Hörassistenzsysteme}

Mit dem Begriff Zubehör für Hörsysteme werden i.d.R. technische Hilfsmittel gemeint, wie z. B. FM-Anlagen, Kinnbügelsysteme zum Fernsehen oder Lichtsignalanlagen. Für viele bedeutet das Wort „Zubehör" dass es sich um einen Gegenstand handelt, der ja nur zusätzlich ist; also dass es auch „ohne“ gehen könnte. Viel besser wäre es, wenn wir den englischsprachigen Begriff Assistive Listening Device (ALD) verwenden, der mit ,assistierenden Hörtechniken" oder Hörassistenzsystemen übersetzt werden kann. Der Begriff der sogenannten Assistenzsysteme hält mittlerweile in verschiedenen Bereichen des täglichen Lebens Einzug, wie z.B. in der Autoindustrie.

Um den Zugang zu einer barrierefreien Kommunikation für Menschen mit einer Hörschädigung zu sichern, ist der Einsatz von Hörassistenzsystemen in Verbindung mit einer Hörsystemversorgung unverzichtbar. Doch ist es den meisten Menschen mit einem Hörsystem gar nicht bewusst, dass es für schwierige Hörsituationen, sei es z. B. beim Telefonieren, beim Fernsehen oder bei Vorträgen eine einfache Lösung gibt!

Deswegen ist eine regelmäßig durchgeführte Bedarfsanalyse und -beratung not-



Abb. 1 Einflüsse auf die Sprachverständlichkeit.

Abb. 2 Prinzip der Hörassistenzsysteme.

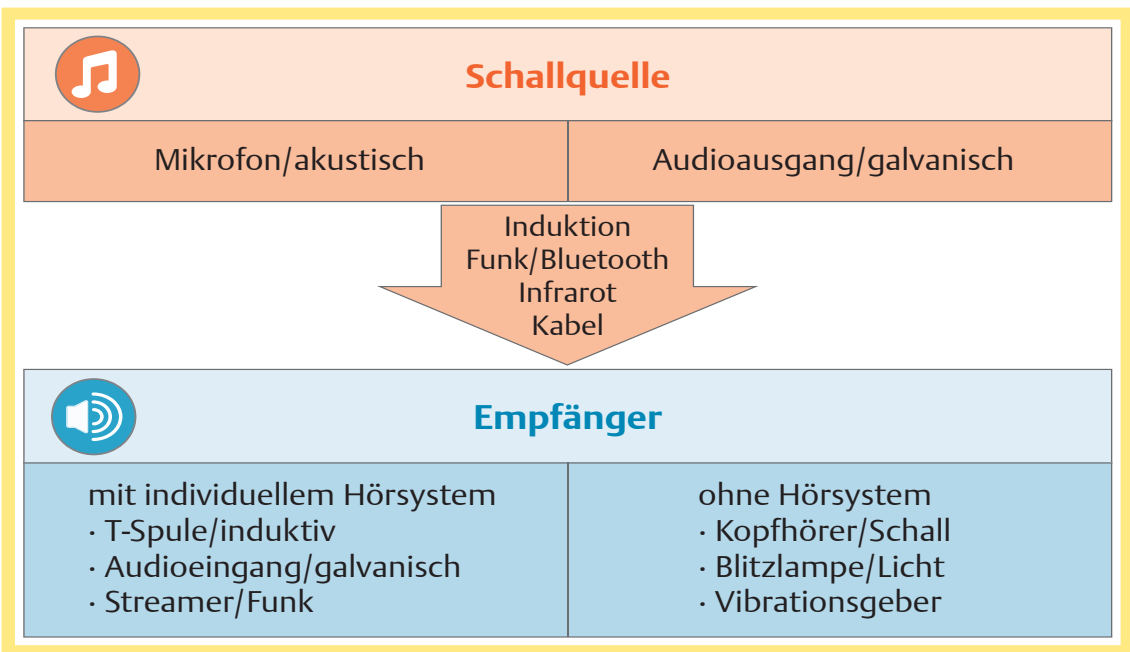

wendig, bei der die zur Verfügung stehenden Lösungen besprochen werden. So kann es eine Lösung sein, die Signalquelle direkt zu beeinflussen, die Raumakustik zu verbessern oder den Störschall zu minimieren. Die Kunst besteht darin, die richtige Auswahl für das individuelle „Hörproblem“ zu finden.

Die Anpassung des Zubehörs auf die individuellen Hörsysteme bzw. dem Hörverlust ist ein weiterer wichtiger Schritt. Für eine langfristige Sicherung des Erfolges schließt sich eine Nachsorge für das Hörassistenzsystem mit regelmäßigen Überprüfungen an. Da sich die Bedürfnisse und
Lebensgewohnheiten ständig ändern, ist dieser Prozess nicht einmalig, sondern als lebenslange Begleitung zu betrachten.

\section{Prinzip der Hörassistenzsysteme $\nabla$}

Die Sprachverständlichkeit wird auf dem Weg vom Sender (der Sprecher, das Telefon, der Fernseher...) zum Empfänger (das Hörsystem) im Wesentlichen von 5 verschiedenen Parametern verschlechtert ( $\bullet$ Abb. 1).

Zur Verbesserung der Kommunikationskompetenz in den individuellen Hör- und Lebenssituationen hilft es vielen Menschen mit einer Hörschädigung, wenn es gelingt, den Schall von der Schallquelle direkt zum Empfänger zu übertragen.

Am Anfang steht die Schallquelle bzw. die Schallaufnahme ( 0 Abb.2). Hier erfolgt die Umwandlung des Schalls in elektrische Signale entweder durch ein Mikrofon oder eine direkte Einspeisung über einen Audioausgang/Klinkenstecker an der TVoder Musikanlage. Liegen die Signale in elektrischer Form vor, kann die Entfernung vom Sender zum Empfänger drahtgebunden mit einem Kabel (unpraktisch) 


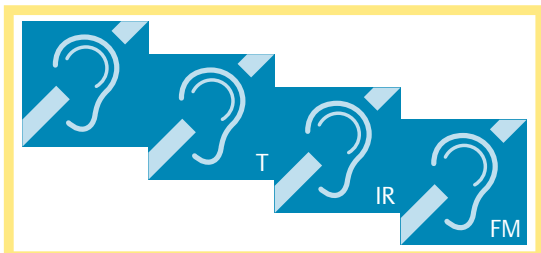

Abb. 3 Hinweiszeichen für die Verfügbarkeit verschiedener Hörassistenzsysteme (T: Induktion, IR: Infrarot; FM für Funk).

oder drahtlos (benötigt Stromversorgung) überbrückt werden. Am sinnvollsten ist eine drahtlose Verbindung, entweder über die Wirkprinzipien Induktion (elektromagnetisches Feld), Infrarot (Licht) oder Funk bzw. Hochfrequenz ( $\bullet$ Abb. 3).

Ist die große Entfernung überbrückt, erfolgt die Ankopplung an den Empfänger, also an das Hörsystem bzw. die Person mit einem Hörverlust. Die Information von der Schallquelle kann dann entweder über die individuellen Hörsysteme oder über einen speziellen Kopfhörer in das Ohr gelangen. Eine Anbindung an die Hörsysteme hat ge- genüber einem Kopfhörer erhebliche Vorteile, wie z.B. den Erhalt des gewohnten Klangbildes oder Ansprechbarkeit für Signale von außen (da das interne Mikrofon noch aktiv sein kann). Somit sollte diese Form bevorzugt werden. Die Signale kommen über mehrere Wege in die Hörsysteme. Dies sind: induktiv über die T-Spule, galvanisch über den Audioeingang oder über Funk direkt bzw. mit einem Zusatzgerät (Streamer).

Weiterhin gibt es die Möglichkeit, Signale auf zusätzlichen Wegen wie Licht für das Auge oder Vibration für die Hautrezeptoren umzuwandeln. Dies ist z.B. für die Wahrnehmung von Warnsignalen von Rauchmeldern oder Weckern für Menschen mit einer Hörschädigung sehr hilfreich.

Durch den Einsatz moderner Technologien ist es mittlerweile möglich, Smartphones/Handys in diesen Prozess einzubinden. Dies ermöglicht Menschen mit einer Hörschädigung auch einen Zugang zur digitalen Welt.

\section{Fazit}

Die Auswahl des „richtigen“ Hörassistenzsystems ist von individuellen technischen Hörgewohnheiten abhängig und benötigt eine fachliche Beratung und Anpassung an eine vorhandene Hörsystemversorgung.

Zur Verbesserung der Kommunikationskompetenz in den individuellen Hörund Lebenssituationen Hörgeschädigter ist der Einsatz von Hörassistenzsystemen zusätzlich zur Hörsystemversorgung unverzichtbar, sie leisten damit einen wichtigen Beitrag für eine „Barrierefreie Kommunikation“.

Dipl.-Ing. Siegrid Meier, Lübeck 